\title{
Nitrogen-containing carbon nanotubes as solid base catalysts $\uparrow$
}

\author{
Stefan van Dommele, Krijn P. de Jong and Johannes H. Bitter* \\ Received (in Cambridge, UK) 17th July 2006, Accepted 12th September 2006 \\ First published as an Advance Article on the web 5th October 2006 \\ DOI: $10.1039 / \mathrm{b} 610208 \mathrm{e}$
}

\begin{abstract}
Nitrogen-containing carbon nanotubes (NCNT) are effective re-usable solid base catalysts, their activity for a Knoevenagel condensation being related to the amount of pyridinic nitrogen incorporated in the NCNT structure, which could be tuned by the synthesis parameters of the catalyst.
\end{abstract}

Nitrogen-containing carbon nanotubes (NCNT) receive much attention because of their potential for use in electronic applications $^{1-5}$ and as gas containers. ${ }^{6}$ Due to the incorporation of nitrogen in the basal planes of the carbon nanotubes (CNT), the physical- and chemical properties of the NCNT are altered as compared to conventional $\mathrm{CNT}^{6-10}$ In this study we have investigated the potential of NCNT for use as base catalysts. Base-catalyzed chemical conversions are key to the production of drugs, fragrances and chemical intermediates ${ }^{11,12}$ in which the use of a regenerable solid base catalyst is prefered over homogeneous bases, like $\mathrm{NaOH}, \mathrm{NH}_{3}$ or $\mathrm{KOCH}_{3}$, since significantly less waste is produced. ${ }^{13-16}$

The NCNT materials, of varying N/C composition, used in this study were prepared by chemical vapour deposition of $\mathrm{C}$ - and $\mathrm{N}$-containing precursors (acetonitrile, pyridine) over a supported metal catalyst, viz. Co or $\mathrm{Ni}$ supported on $\mathrm{SiO}_{2}$ (Table 1). ${ }^{17,18}$ The growth catalyst was chemically removed after NCNT production. The relation between the type of basic N-species in the NCNT and the catalytic activity for the Knoevenagel condensation (Scheme 1) of ethyl- $\alpha$-cyanoacetate (ECA) with benzaldehyde (BA) has been studied and established.

The morphology of the NCNT was examined by Transmission Electron Microscopy (TEM) using a Tecnai20FEG electron microscope. Fig. 1 displays both medium and high resolution TEM micrographs of a typical NCNT sample. Uniform NCNT were observed with an average tube diameter of $25 \mathrm{~nm}$. The medium magnification micrograph displayed a significant contrast between the centre and edge of the material suggesting tube

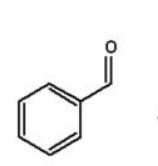

benzaldehyde

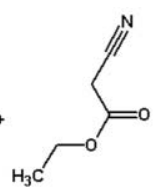

ethylcyanoacetate
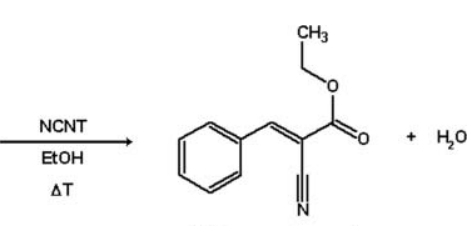

ethyl-a-cyanocinnamate
Scheme 1 Knoevenagel condensation; benzaldehyde reacts with ethylcyanoacetate to form ethyl- $\alpha$-cyanocinnamate.

Utrecht University, Inorganic Chemistry and Catalysis, Sorbonnelaan 16, Utrecht, The Netherlands.E-mail:J.H.Bitter@chem.uu.nl,

Fax: +31 30251 1027; Tel: +31302537400

$\uparrow$ Electronic supplementary information (ESI) available: Summary and references. See DOI: 10.1039/b610208e morphology and an on-top view (examples indicated with arrows) further proved that the material comprised hollow tubes. High resolution TEM (Fig. 1, inset) showed that the prepared materials consisted of multiwall tubes with an average graphene sheet spacing of $3.4 \AA$. Some physicochemical properties of the samples have been compiled in Table 1 .

The N/C atomic ratio in the NCNT was determined by X-ray Photoelectron Spectroscopy (XPS) using a Vacuum Generators XPS system operating with $\mathrm{Al}(\mathrm{K} \alpha)$ radiation. The amount of nitrogen incorporated in the NCNT (Table 1) varied between 7.4 and $3.6 \mathrm{mmol} \mathrm{g}^{-1} \mathrm{NCNT}$ and decreased with increasing growth temperature for a given growth catalyst and $\mathrm{C} / \mathrm{N}$ source. This might be explained by the impact of the growth temperature on one of the three stages that are envisaged during NCNT growth, viz. (1) decomposition of the precursor to surface carbon, hydrogen and nitrogen atoms, (2) diffusion/migration of these atoms through/over the metal and (3) formation of the NCNT. ${ }^{19}$

In step 1, i.e. the decomposition of the precursor, a recombination and desorption of the surface $\mathrm{N} / \mathrm{H}$ atoms as $\mathrm{N}_{2}$ or $\mathrm{NH}_{3}$ might occur which was confirmed by a qualitative analysis of the gas phase by mass spectrometry. It can be speculated that this parallel process is more significant at high temperatures, resulting in a lower amount of $\mathrm{N}$ in the NCNT.

Different types of nitrogen species in the graphene sheets, i.e., pyridynic, quaternary, pyrrolic and $\mathrm{N}$-oxides were discriminated by means of XPS. Analysis of the N1s signal revealed that in our samples the majority of the nitrogen was present as pyridinic(binding energy $398.6 \mathrm{eV}$ ) and quaternary nitrogen (binding energy $401.4 \mathrm{eV}$ ). The former is located at the edges of the graphene sheets or at defects in the plane while the latter is located within the sheets i.e., carbon is substituted by nitrogen. ${ }^{20,21}$ The amount of pyridinic and quaternary nitrogen could be tuned by the synthesis temperature. At low temperature, i.e. $823 \mathrm{~K}$, the formation of pyridinic nitrogen was favoured while at higher temperatures the formation of quaternary nitrogen was predominant (Table 1). This is in line with earlier observations that pyridinic nitrogen in coals can be converted to quaternary ones by a high temperature treatment. ${ }^{22-24}$

The basic properties of the NCNT, i.e. the number and strength of accessible basic sites, were determined with acid-base titration using a Titralab Tim880 setup. About $0.1 \mathrm{~g}$ of the material was degassed in vacuum at $423 \mathrm{~K}$ for $1 \mathrm{~h}$ and then suspended in $50 \mathrm{~mL}$ decarbonized $0.1 \mathrm{M}$ potassium chloride solution under a nitrogen atmosphere. Next, the suspension was titrated with a $2 \times 10^{-3} \mathrm{M}$ hydrochloric acid solution and the $\mathrm{pH}$ was recorded as a function of the volume of titrant added. The titration curves of selected NCNT are displayed in Fig. 2. The initial $\mathrm{pH}$ of all samples suspended in water was 8-9, which provides direct evidence that all NCNT were mildly basic. The evolution of the $\mathrm{pH}$ as a function of 
Table 1 Some properties of the NCNT

\begin{tabular}{|c|c|c|c|c|c|c|c|}
\hline Sample & $\mathrm{C} / \mathrm{N}$ source & $T / \mathrm{K}$ & $\begin{array}{l}\text { Yield } / \mathrm{g} \mathrm{g}^{-1} \\
\text { metal }\end{array}$ & $\begin{array}{l}N_{\text {total }}(\mathrm{XPS})^{a} / \\
\mathrm{mmol} \mathrm{g}^{-1} \mathrm{NCNT}\end{array}$ & $\begin{array}{l}N_{\text {total }}(\text { titration })^{b} / \\
\text { mmol g }{ }^{-1} \text { N-CNT }\end{array}$ & $N_{\mathrm{P}} / N_{\mathrm{Q}}{ }^{c}$ & $N_{\mathrm{P}} / N_{\mathrm{T}}^{d}$ \\
\hline Co823ACN & Acetonitrile & 823 & 10 & 7.4 & 0.033 & 2.0 & 1.0 \\
\hline Co923ACN & Acetonitrile & 923 & 17 & 4.0 & 0.029 & 1.0 & 0.6 \\
\hline Co1023ACN & Acetonitrile & 1023 & 25 & 4.3 & 0.021 & 0.6 & 0.4 \\
\hline Co1023PYR & Pyridine & 1023 & 6 & 7.4 & 0.024 & 1.5 & 0.7 \\
\hline Ni823ACN & Acetonitrile & 823 & 10 & 5.0 & 0.027 & 1.7 & 0.9 \\
\hline Ni1023ACN & Acetonitrile & 1023 & 6 & 3.6 & 0.013 & 0.6 & 0.3 \\
\hline
\end{tabular}

${ }^{a}$ Total amount of nitrogen in NCNT determined with XPS from C1s and N1s areas. ${ }^{b}$ Total amount of accessible sites determined with acidbase titration. ${ }^{c} N_{\mathrm{P}}=$ pyridinic $\mathrm{N}, N_{\mathrm{Q}}=$ quaternary N. ${ }^{d}$ Ratio of pyridinic nitrogen to total $\mathrm{N}$ species.

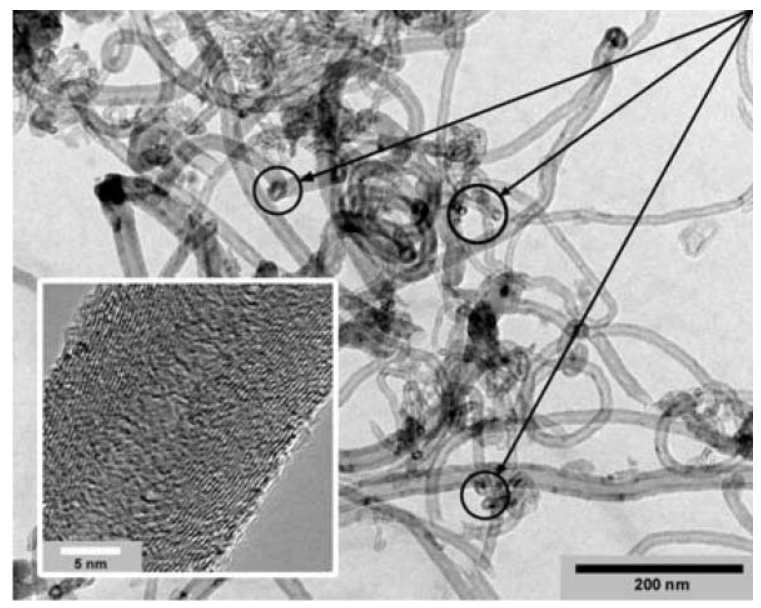

Fig. 1 TEM images of Co923ACN. Arrows indicate the on-top view of the tubes. The inset is a HRTEM image of a NCNT showing parallel graphene layers.

the added volume of titrant varied with the NCNT preparation procedure indicating differences in the number and strength of basic sites incorporated in the materials. The amounts of accessible basic sites were calculated from the amount of $\mathrm{HCl}$ added to obtain a $\mathrm{pH}$ of 7 and have been compiled in Table 1. Surprisingly, the amount of basic sites determined with titration was about two orders of magnitude lower compared to the amount of nitrogen determined by XPS. We speculate that the majority of the nitrogen

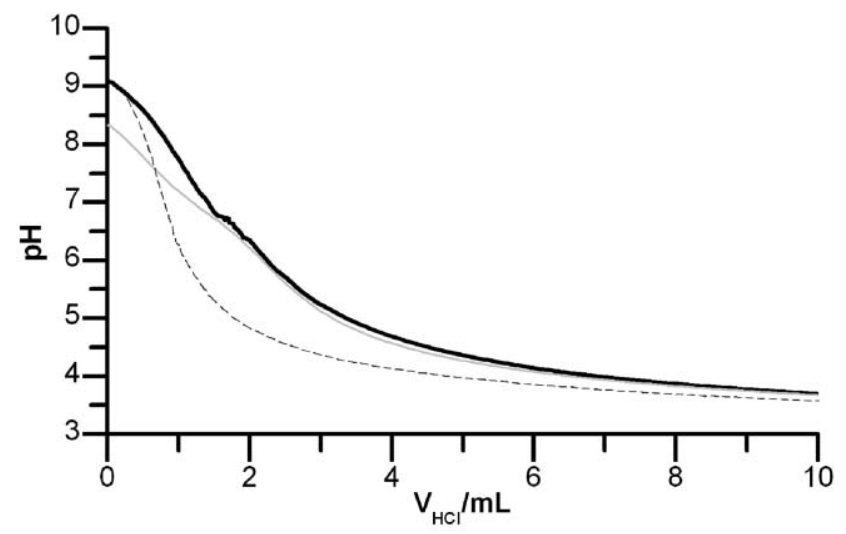

Fig. 2 Titration curves of NCNT grown from acetonitrile over Co: Co823ACN (black line), Co923ACN (grey line) and Co1023ACN (dashed line). incorporated in the NCNT are located in graphene layers below the NCNT surface which are not accessible for the acid and can therefore not be detected with titration.

The catalytic activity of the NCNT was tested in the Knoevenagel condensation of benzaldehyde (BA) with ethylcyanoacetate (ECA) to form ethyl- $\alpha$-cyanocinnamate (ECC). The NCNT $(1 \mathrm{~g})$ were heated to $423 \mathrm{~K}$ under vacuum and kept at that temperature for $1 \mathrm{~h}$. All further handling was carried out in a nitrogen atmosphere. Freshly distilled BA $\left(1 \times 10^{-2} \mathrm{~mol}\right)$ and ECA $\left(1 \times 10^{-2} \mathrm{~mol}\right)$ were dissolved in $50 \mathrm{~mL}$ ethanol (p.a. grade) and pretreated to remove any dissolved carbon dioxide using the freeze-thaw method. Subsequently, the reaction mixture was added to the NCNT and the suspension was stirred vigorously and heated to reflux temperature. Samples of the reaction mixture were taken regularly for GC analysis. For reference, a blank reaction of only BA and ECA was performed. The activity of the blank was always subtracted from the measured activity with catalyst. Also non-functionalized Carbon Nanofibers (CNF) were tested for reference purposes in the same manner as NCNT. In Fig. 3 the initial activity, corrected for blank activity, for the Knoevenagel condensation has been plotted as a function of the amount of pyridinic N. As can be deduced from this figure, all NCNT samples displayed activity for this reaction. The product yields were between 20 and $50 \%$ after $1 \mathrm{~h}$. Please note that the nonfunctionalized carbon nanofiber sample did not show any activity in the condensation, indicating that indeed the incorporated nitrogen is responsible for the observed activity of NCNT. The NCNT which showed a high conversion in the condensation reaction, Co823ACN, was after filtration and washing used in a second run. The conversion was similar to that of the first run thereby demonstrating its reusability.

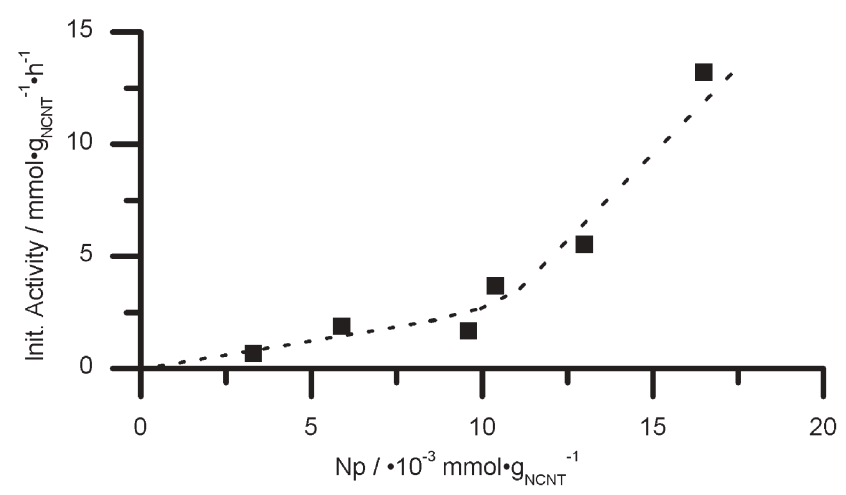

Fig. 3 Relation of pyridinic nitrogen and initial activity. 
For further study of the relation between the catalyst's activity and the type of nitrogen present, the pyridinic/total $\mathrm{N}$ ratio from XPS (Table 1) together with the amount of base sites determined from titration, were used to calculate the amount of each nitrogen type at the NCNT. Please note that it was thus assumed that the relative distribution of each nitrogen type as determined by XPS is valid for the accessible surface of the NCNT. The initial activity in the Knoevenagel condensation increased with increasing amount of pyridinic nitrogen, which indicated that this type of nitrogen is (part of) the active site for the reaction. No relation could be found between the quaternary nitrogen species and activity. Since all NCNT samples obeyed this relation irrespective of the nitrogen source, growth catalyst and growth temperature, it was concluded that the route to the final NCNT was not critical.

To appreciate the activity of the NCNT materials, the initial activities per gram of catalyst have been compared with the reported activities of other mildly basic materials like fluoro- and hydroxyappatites and aluminophosphate oxynitrides. ${ }^{25,26}$ It turned out that our materials exhibit activities similar to these basic catalysts. Yields between $0.2 \times 10^{-3}$ and $20.8 \times 10^{-3} \mathrm{~mol}$ product $\mathrm{h}^{-1} \mathrm{~g}^{-1}$ catalyst were reported for the fluoro- and hydroxyappatites and aluminophosphate oxynitrides. Hydrotalcite based materials and functionalized MCM displayed higher yields around $40 \times 10^{-3}$ mol product $\mathrm{h}^{-1} \mathrm{~g}^{-1}$ catalyst. ${ }^{27,28}$ With yields between $2 \times 10^{-3}$ and $8 \times 10^{-3}$ mol product the activity of our materials is in a similar range as those of the mild solid bases such as fluoro- and hydroxyappatites and aluminophosphate oxynitrides. It must be mentioned that the catalytic data from the literature was obtained at a variety of conditions. Therefore only the order of magnitudes of activities can be compared.

To conclude we have demonstrated that nitrogen-containing carbon nanotubes display basic properties and can be used as solid base catalysts. The catalytic activity of the samples seemed to be determined by the concentration of pyridinic nitrogen present in the catalyst. These materials provide new opportunities for welldefined solid base catalysts.

This investigation is supported by The Netherlands Organization for Scientific Research, project 700.50.520.

\section{Notes and references}

1 C. P. Ewels and M. Glerup, J. Nanosci. Nanotechnol., 2005, 5(9), 1345.

2 M. Terrones, N. Grobert and H. Terrones, Carbon, 2002, 40, 1665.

3 X. Wang, Y. Liu, D. Zhu, L. Zhang, H. Ma, N. Yao and B. Zhang, J. Phys. Chem. B, 2002, 106, 2186.

4 R. Kurt, J. M. Bonard and A. Karimi, Thin Solid Films, 2001, 193, 398.

5 P. Ding, E. Liang, M. Chao, X. Chuo and J. Zhang, Physica E, 2005, 25, 654 .

6 M. Terrones, R. Kamalakaran, T. Seeger and M. Rühle, Chem. Commun., 2000, 2335.

7 C. Tang, D. Goldberg, Y. Bando, F. Xu and B. Liu, Chem. Commun., 2003, 3050

8 S. H. Lim, H. I. Elim, X. Y. Gao, A. T. S. Wee, W. Ji, J. Y. Lee and J. Lin, Phys. Rev. B: Condens. Matter, 2006, 73, 45402.

9 R. Czerw, M. Terrones, J. C. Charlier, X. Blasé, B. Foley, R. Kamalakaran, N. Grobert, H. Terrones, D. Tekleab, P. M. Ajayan, W. Blau, M. Rühle and D. L. Carroll, Nano Lett., 2001, 1(9), 457.

10 S. Souto, M. Pickholz, M. C. dos Santos and F. Alvarez, Phys. Rev. B. Condens. Matter, 1998, 57(4), 2536.

11 G. J. Kelly, F. King and M. Kett, Green Chem., 2002, 4, 392.

12 K. Tanabe and W. F. Hölderich, Appl. Catal., A, 1999, 181, 399.

13 R. A. Sheldon, J. Mol. Catal. A: Chem., 1996, 107, 75.

14 H. U. Blaser, Catal. Today, 2000, 60, 161.

15 F. King and G. J. Kelly, Catal. Today, 2002, 73, 75.

16 F. Winter, V. Koot, A. J. van Dillen, J. W. Geus and K. P. de Jong, J. Catal., 2005, 236, 91

17 M. Nath, B. C. Satishkumar, A. Govindaraj, C. P. Vinod and C. N. R. Rao, Chem. Phys. Lett., 2000, 322, 333.

18 C. Tang, Y. Bando, D. Goldberg and F. Xu, Carbon, 2004, 42, 2625.

19 K. P. de Jong and J. W. Geus, Catal. Rev. Sci. Eng., 2000, 42(4), 481.

20 F. Kapteijn, J. A. Moulein, S. Matzner and H. P. Boehm, Carbon, 1999, 37, 1143.

21 K. Stanczyk, R. Dziembaj, Z. Piwowarska and S. Witkowski, Carbon, 1995, 33(10), 1383.

22 J. R. Pels, J. A. Moulijn, Q. Zhu and K. M. Thomas, Carbon, 1995, 33, 1641.

23 H. C. Choi, S. Y. Bae, W. S. Jang and J. Park, J. Phys. Chem. B, 2005, 109, 1683.

24 I. Shimoyama, G. Wu, T. Sekiguchi and Y. Baba, J. Electron Spectrosc. Relat. Phenom., 2001, 114-116, 841.

25 N. Elazarifi, A. Ezzamarty, J. Leglise, L.-C. de Ménorval and C. Moreau, Appl. Catal., A, 2004, 267, 235.

26 A. Massinon, J. A. Odriozola, Ph. Bastians, R. Conanec, R. Marchand, Y. Laurent and P. Grange, Appl. Catal., A, 1996, 137, 9.

27 M. L. Kantam, B. M. Choudary, Ch. V. Reddy, K. K. Rao and F. Figueras, Chem. Commun., 1998, 1033.

28 I. Rodriguez, S. Iborra, A. Corma, F. Rey and J. L. Jordá, Chem. Commun., 1999, 593. 